\title{
Malaria Treatment in Children Based on Presumptive Diagnosis: A Make or Mar?
}

\section{Chukwuocha UM*}

Department of Public Health, Federal University of Technology, Nigeria

*Corresponding author: Chukwuocha UM, Department of Public Health, Federal University of Technology, Nigeria, E-mail: uchukwuocha@gmail.com

Received date: February 23, 2016; Accepted date: March 17, 2016; Published date: March 21, 2016

Citation: Chukwuocha UM (2016) Malaria Treatment in Children Based on Presumptive Diagnosis: A Make or Mar?. Pediatric Infect Dis 1: 6. doi: $10.21767 / 2573-0282.100006$

Copyright: ${ }^{\odot}$ 2016, Chukwuocha UM. This is an open-access article distributed under the terms of the Creative Commons Attribution License, which permits unrestricted use, distribution, and reproduction in any medium, provided the original author and source are credited.

Keywords: Malaria; Malaria treatment; Children

\section{Commentary}

Presumptive Diagnosis and Treatment of malaria in endemic areas has been a subject of intense debate. The effectiveness of this strategy is hindered by the prevalence of other febrile illnesses with symptoms that overlap with that of malaria. Despite, the World Health Organization's (WHO) recent recommendation that malaria should be confirmed with specific tests before treatment, the practice is still very common. This short communication discusses the current situation considering the WHO guidelines with recommendations on its optimization and sustainability.

Malaria, an illness caused by the protozoa, plasmodium is conventionally diagnosed by microscopic examination of stained blood films. However because of the high morbidity and mortality rates recorded due to malaria especially among children in endemic areas as a result of treatment delays, treatment following presumptive diagnosis was advocated and recommended. Presumptive diagnosis implies diagnosing the illness based on symptoms associated with the illness without other confirmatory tests. This method of diagnosis gained a lot of popularity because most presumed malaria treatments in endemic areas especially in Sub Saharan Africa were already done at home. In this case every fever is almost entirely attributed to malaria illness in these areas and is used in diagnosis at home. Fever can be defined as a body temperature that is above the normal range resulting from an increase in the body's temperature set-point [1,2]. Many sources define it as values between 37.5 and $38.3^{\circ} \mathrm{C}\left(99.5\right.$ and $\left.100.9{ }^{\circ} \mathrm{F}\right)$ [1]. Other symptoms that are also used in presumptive diagnosis include headache, chills, vomiting, and pains in parts of the body, anorexia and general body weakness [3]. The combination of all or some of these symptoms had been used to create an algorithm for the presumptive or clinical diagnosis of malaria [4-7]. However other illnesses exist that mimic these set of symptoms especially fever. These illnesses are collectively called febrile illnesses. Some febrile illnesses also prevalent in malaria endemic areas with significant morbidity and mortality include meningitis, hepatitis, typhoid as well as other illnesses of unknown aetiology [8-10].
Malaria remains the most devastating infectious disease in the tropical regions of the World imposing a vast burden of mortality and perpetuating cycles of poverty especially in rural endemic communities. According to the World Malaria Report, an estimated 3.3 billion people in 97 countries and territories are at risk of malaria, and 1.2 billion are at high risk ( $>1$ case of malaria per 1000 population each year) [11]. Malaria is concentrated in low-income and lower income countries where those mostly affected live in the poorest and most marginalized communities [11]. Such communities have the highest risks associated with malaria, and the least access to effective services for prevention, diagnosis and treatment [12]. Several concerted efforts made towards its control have so far not yielded the desired results in such areas. The burden is further compounded by other febrile illnesses with symptoms that overlap those of malaria.

Despite the fact that the main strategy for reducing childhood mortality and morbidity is presumptive treatment of all fevers in children with antimalarial drugs, the greatest challenge of malaria treatment still occurs during treatment as many children with malaria are treated at home [13]. Presumptive diagnosis and treatment which would have been very effective in curtailing the burden of this disease complicates the burden due to over use or misuse of antimalarial therapies. The resultant effect is increased malaria parasite resistance and mistreatment of other non-malarial febrile illnesses. Apart from the risk of malaria parasite resistance and increased morbidity and mortality of other mistreated illnesses, there is also an increased cost in seeking treatment when the misdiagnosed illness fully manifests.

Factors that encourage presumptive diagnosis and treatment at the home front may be attributed to lack of appropriate education and information as well as lack of access to adequate diagnosis and the cost associated with it $[14,15]$. Increased reliance on advice from relatives and community medicine vendors is also another factor. The high endemicity of malaria, perceived delay in microscopy results and impression of incompetence created on reliance of diagnostic results before treatment have been suggested as some of the reasons why health providers practice presumptive treatment of childhood malaria [16]. Other reasons may include non-availability of 
diagnostic test kits (RDTs) in the consulting rooms and doubt about their reliability.

The World Health Organization however swiftly introduced new guidelines on the treatment of malaria which states that, whenever possible, "in all settings, clinical suspicion of malaria should be confirmed with a parasitological diagnosis" [17]. The current guideline has changed previous stand on presumptive treatment of all childhood fever in sub-Saharan Africa regions with anti-malarial drugs. This also recognizes the urgent need for new, simple, quick, accurate, and cost-effective diagnostic tests for determining the presence of malaria parasites. This will aid in overcoming the deficiencies of light microscopy by developing numerous new malaria-diagnostic techniques. In this regard, the WHO's test and treat strategy includes the introduction and deployment of malaria rapid diagnostic test kits (RDTs) to health facilities. This is intended to make the practice of presumptive treatment less fashionable particularly in tertiary hospitals.

It is however surprising that even with the introduction of these new guidelines, the expected improvement in the practice of confirmation of malaria prior to treatment is not significantly reported [18]. In fact, presumptive diagnosis and treatment of malaria is still being practiced not only in the homes but in health care facilities including the tertiary health care facilities. This therefore means that there may be more to the continued practice of presumptive treatment of malaria than the presumed difficulties with microscopy. The continued practice of presumptive treatment without adequate confirmation may only lead to increased complexity and burden of febrile illnesses.

The benefits of confirmed diagnosis and treatment of malaria in endemic areas include but not limited to reduction of drug wastage, avoidance of increased parasite resistance and avoidance of adverse drug events. It also guarantees the evasion of imminent loss of confidence in anti-malarial drugs by the public due to use of anti-malaria's in cases that are not malaria cases.

In other to save the current situation therefore, it is very important that constant community health education is put in place to educate especially the local people on recommended guidelines for the management of malaria and other febrile illnesses. There is needed to make sure that community epidemiological evidence based data are always updated and available. Adequate mechanisms should be put in place to monitor and evaluate the practice of guidelines on management of febrile illnesses both at home and in health facilities. Furthermore, local authorities should be encouraged to establish community diagnostic centres where trained community health workers can aid in quickly confirming febrile illnesses at the community level. Cost effective RDTs and other diagnostic tools should also be made available for easy accessibility and affordability. Continuous evaluation of the RDTs should be carried out to make sure that the rates of false results are significantly reduced. Research should focus more on finding some therapeutics that can concomitantly treat some of the febrile illnesses. The development of whole plant therapies with their complex chemical structure and constituents may be very important in this regard. Finally, people from communities where malaria and other infectious diseases are prevalent should be empowered to be able to adequately and sustainably manage and control these diseases by themselves. They should be considered and involved when policies are being made and implemented.

\section{References}

1. O'Neill MB (1994) Fever in Children Canadian Journal of Paediatrics 2: 48-49.

2. Wright PF, McKee KT, Sell SH (1981) Patterns of illness in the highly febrile young child: epidemiologic, clinical, and laboratory correlates. Pediatrics 67: 694-700.

3. Radetsky M (1984) The clinical evaluation of the febrile infant. Primary Care 11: 395-405.

4. Mwangi TW, Mohammed M, Dayo H, Snow RW, Marsh K (2005) Clinical algorithms for malaria diagnosis lack utility among people of different age groups. Tropical Medicine and International Health 10: 530-536.

5. Chandramohan D, Jaffar S, Greenwood B (2002) Use of clinical algorithms for diagnosing malaria. Tropical Medicine and International Health 7: 45-52.

6. Weber MW, Mulholland EK, Jaffar S, Troedsson H, Gove $\mathrm{S}$ Greenwood BM (1997) Evaluation of an algorithm for the integrated management of childhood illness in an area with seasonal malaria in the Gambia. Bulletin of the World Health Organization 75: 25-32.

7. Tarimo DS, Minjas JN, Bygbjerg IC (2001) Malaria diagnosis and treatment under the strategy of the integrated management of children illness (IMCI): relevance of laboratory support from the rapid immunochromatographic tests of ICT malaria P.f/P.v and OptiMAL. Annals of Tropical Medicine and Parasitology 95: 437-444.

8. Planche $T$, Agbenyega $T$, Bedu-Addo G, Ansong D, Owusu-Ofori A, et al. (2003) A prospective comparison of malaria with other severe diseases in African children: prognosis and optimization of management. Clinical Infectious Diseases 37: 890-897.

9. Nankabirwa J, Zurovac D, Njogu JN, Rwakimari JB, Counihan H, et al. (2009) Malaria misdiagnosis in Uganda-implications for policy change. Malaria Journal 8: 66.

10. A-Elgayuom SM, El-Feki Ael K, Mahgoub BA, El-Rayah el A, Giha HA (2009) Malaria overdiagnosis and burden of malaria misdiagnosis in the suburbs of central Sudan: special emphasis on artemisininbased combination therapy era. Diagnostic Microbioliology and Infectious Diseases 64: 20-26

11. World Health Organization (2014) World Malaria Report, Geneva, Switzerland.

12. Omumbo JA, Noor AM, Fall IS, Snow RW (2013) How Well Are Malaria Maps Used to Design and Finance Malaria Control in Africa? PLOS one 8: e53198

13. Breman JG (2001) The ears of the hippopotamus: manifestations, determinants, and estimates of the malaria burden. American Journal of Tropical Medicine and Hygiene 64: 1-11

14. Chukwuocha UM (2001) Rapid assessment of home management of malaria among caregivers in parts of South Eastern Nigeria. Pan African Medical Journal 10: 29.

15. Chukwuocha UM, Nwakwuo GC, Emerole C, Dozie INS, Nwuda OEC (2014) Prevalent home management techniques and 
outcome among mothers of febrile children in Eastern Nigeria. Journal of Public Health and Epidemiology 6: 111-118.

16. Ughasoro MD Okoli CC, Uzochukwu BSC (2013) Qualitative study of presumptive treatment of childhood malaria in third tier tertiary hospitals in southeast Nigeria: a focus group and in-depth study. Malaria Journal 12: 436

17. World Health Organization (2005) Technical Updates of the Guidelines on the Integrated Management of Childhood Illness (IMCI).
18. Graz B, Willcox M, Szeless T, Rougemont T (2011) Test and treat or presumptive treatment for malaria in high transmission situations? A reflection on the latest WHO guidelines. Malaria Journal 10: 136. 